\title{
Nanoscale
}

Check for updates

Cite this: Nanoscale, 2022, 14, 626

Received 22nd October 2021,

Accepted 13th December 2021

DOI: $10.1039 / \mathrm{d} 1 \mathrm{nr} 06998 \mathrm{e}$

rsc.li/nanoscale

\section{Fast and versatile thermo-osmotic flows with a pinch of salt $\uparrow$}

\author{
Cecilia Herrero, (DD a Michael De San Féliciano, ${ }^{a}$ Samy Merabia (DD *a and \\ Laurent Joly iD *a,b
}

\begin{abstract}
Thermo-osmotic flows - flows generated in micro and nanofluidic systems by thermal gradients - could provide an alternative approach to harvest waste heat. However, such use would require massive thermo-osmotic flows, which are up to now only predicted for special and expensive materials. Thus, there is an urgent need to design affordable nanofluidic systems displaying large thermo-osmotic coefficients. In this paper, we propose a general model for thermo-osmosis of aqueous electrolytes in charged nanofluidic channels, taking into account hydrodynamic slip, together with the different solvent and solute contributions to the thermo-osmotic response. We apply this model to a wide range of systems by studying the effects of wetting, salt type and concentration, and surface charge. We show that intense thermo-osmotic flows can be generated using slipping charged surfaces. We also predict for intermediate wettings a transition from a thermophobic to a thermophilic behavior depending on the surface charge and salt concentration. Overall, this theoretical framework opens an avenue for controlling and manipulating thermally induced flows with common charged surfaces and a pinch of salt.
\end{abstract}

Due to the increasing world energy consumption and the need for new clean energies, waste heat harvesting is a major challenge for decades to come. Some of the most common difficulties in harvesting waste heat come from the small temperature differences between the source and the environment $\left(<50{ }^{\circ} \mathrm{C}\right)^{1}$ as well as from the need to use rare, expensive and often toxic thermoelectric materials. ${ }^{2}$ Alternatively, thermo-osmotic flows (generated at liquid-solid interfaces by temperature gradients) can be used to transform waste heat into electricity via a turbine $^{3}$ or to pump water for desalination. ${ }^{4,5}$ Thermo-osmotic flows also directly generate electric current on charged surfaces ${ }^{6,7}$

\footnotetext{
${ }^{a}$ Univ Lyon, Univ Claude Bernard Lyon 1, CNRS, Institut Lumière Matière, F-69622 Villeurbanne, France. E-mail: laurent.joly@univ-lyon1.fr, samy.merabia@univ-lyon1.fr

${ }^{b}$ Institut Universitaire de France (IUF), 1 rue Descartes, 75005 Paris, France $\dagger$ Electronic supplementary information (ESI) available: Analytical framework derivation; molecular dynamics simulation details; discussion on the generality of the proposed model; thermo-osmotic response results for all wettings. See DOI: $10.1039 / \mathrm{d} 1 \mathrm{nr} 06998 \mathrm{e}$
}

by advecting the charge of the electric double layer (EDL) appearing in the liquid to screen the surface charge. ${ }^{8}$ Historically, the first experimental results on thermo-osmosis were published by Lippmann ${ }^{9}$ and Aubert ${ }^{10}$ at the beginning of the 20th century. Since then, a broad literature has been devoted to the study of aqueous solutions and various membranes, both from experiments $^{11-15}$ or molecular dynamics (MD) simulations. ${ }^{16-20}$ Some disagreements have been reported for aqueous electrolytes, specifically in the flow direction (toward the hot side, so-called thermophilic flow, or toward the cold side, so-called thermophobic flow) for similar systems, ${ }^{21-24}$ or on the relation between the flow amplitude and surface charge. ${ }^{13}$ Although with exceptions, a thermophobic flow is generally expected for hydrophobic membranes and a thermophilic flow for hydrophilic membranes. ${ }^{25,26}$ Such differences cannot be understood by the classical theory ${ }^{27}$ developed by Derjaguin and Sidorenkov ${ }^{11,28}$ and by Ruckenstein for thermophoresis. ${ }^{29}$ This theory, based on the electrostatic enthalpy of the EDL, predicts that the flow is controlled by the electric surface charge, and always goes to the hot side. Other authors have recently proposed a different understanding in terms of irreversible thermodynamics by taking into account solvent contribution. ${ }^{15}$ However, their description only explains qualitatively the flow direction and does not provide a microscopic description of the effects of fluid wetting properties, salt concentration and electric surface charge.

Thermo-osmosis has seen a renewed interest due to the massive thermo-osmotic responses predicted by the use of novel materials, such as soft nanochannels, ${ }^{30}$ carbonnanotubes ${ }^{5,18,31}$ or graphene, ${ }^{17}$ together with novel experiments by Bregulla et al. ${ }^{24}$ who first reported a microscale manifestation of thermo-osmotic flows. Thermo-osmotic flows could, in particular, be boosted by the slip boundary condition (BC) that describes the velocity jump $v_{\mathrm{s}}$ at the interface by a general expression first proposed by Navier: ${ }^{32,33}$

$$
v_{\mathrm{s}}=\left.b \frac{\partial v}{\partial z}\right|_{z=z_{\mathrm{s}}}
$$

where $z_{\mathrm{s}}$ corresponds to the shear plane position ${ }^{34}$ and $b$ denotes the slip length. ${ }^{35}$ The role of interfacial hydrodyn- 
amics in thermo-osmosis has already been explored in the literature. ${ }^{16,17,36}$ Furthermore, in recent works on thermo-electricity, the critical role of solvent enthalpy in describing the response has been highlighted for a model, highly hydrophobic surface. ${ }^{7}$

Following this work, we propose in this communication an analytical framework with the objective to predict thermoosmosis of aqueous electrolytes confined by charged surfaces, extendable to thermoelectricity and thermophoresis. Solvent contribution and electrostatic ionic interactions are shown to play the leading role along with hydrodynamic slip. We apply the model to a wide range of systems by varying the wetting interaction, salt type and concentration, and surface charge. We report large thermo-osmotic responses, comparable to the highest responses predicted for special systems as inferred from previous simulations, ${ }^{5,16,17,31}$ as well as a change of sign in the flow direction. Such a change of sign cannot be predicted by only considering electrostatic interactions and can be crucial in order to interpret the different experimental results reported in the literature.

\section{Theoretical framework}

The thermo-osmotic response of a liquid-solid interface is quantified by the thermo-osmotic coefficient $M_{\text {to }}$, defined from the relation $v_{\text {to }}=M_{\text {to }}(-\nabla T / T)$, where $\nabla T / T$ is the relative temperature gradient parallel to the wall and $v_{\text {to }}$ is the generated thermo-osmotic velocity in the bulk region (where the liquid does not interact with the wall). ${ }^{27}$ In ref. 17, the authors propose a modification to the classical Derjaguin theory ${ }^{28}$ and show that, in order to take into account the hydrodynamic BC, the thermo-osmotic response coefficient can be expressed as (see the ESI $\dagger$ ):

$$
M_{\mathrm{to}}=\frac{1}{\eta} \int_{z_{0}}^{\infty}\left(z-z_{\mathrm{s}}+b\right) \delta h(z) \mathrm{d} z,
$$

where $\eta$ is the liquid viscosity, $z$ is the distance to the surface, $\delta h$ is the enthalpy excess density, $b$ is the slip length defined from eqn (1), and $z_{\mathrm{s}}$ corresponds to the shear plane position. One can account for the presence of slip or a stagnant layer close to the wall by introducing an effective slip length $b_{\text {eff }}=$ $b-z_{\text {s }}$ (see Fig. 1a). When $b_{\text {eff }} \geq 0$ (slip situation), the velocity profile does not vanish in the water slab and therefore the integral in eqn (2) should be performed from the wall position considered at zero, $z_{0}=0$. On the contrary, if $b_{\text {eff }}<0$ (stagnant layer situation), then $b_{\text {eff }}$ identifies with the size of a stagnant layer present at the liquid-solid interface, where the liquid velocity vanishes. In this case, the stagnant layer does not contribute to the integral in eqn (2) and consequently $z_{0}=-b_{\text {eff }}$.

With regard to the enthalpy excess density $\delta h$, the standard approach $^{28,29}$ assumes that it is mostly determined by the electrostatic enthalpy of ions in the EDL, $\delta h_{\mathrm{el}}(z)=\rho_{\mathrm{e}}(z) V(z)+$ $p(z)$, where $\rho_{\mathrm{e}}$ is the charge density, $V$ is the local electric potential and $p$ is the pressure. Using the Poisson equation $\rho_{\mathrm{e}}=$ $-\varepsilon d_{z}^{2} V$ (assuming a constant solvent permittivity $\varepsilon$ ) and con-
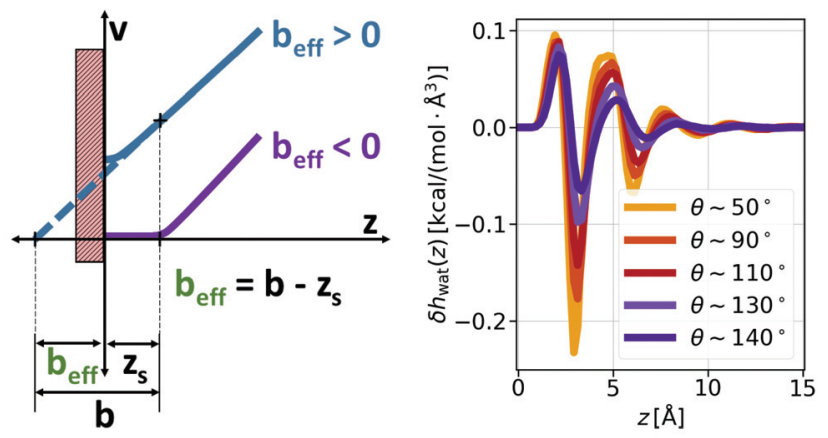

(a)

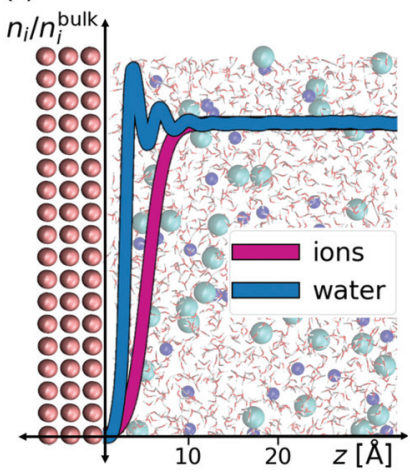

(b)

Fig. 1 (a) Schematics of the effective slip length $b_{\text {eff }}$ as a function of the slip length $b$ and the shear plane position $z_{s}$. We distinguish between the slip situation $\left(b_{\text {eff }}>0\right)$ and the stagnant layer situation $\left(b_{\text {eff }}<0\right)$. (b) Modelled system for the measures of water enthalpy excess density and slip, together with the normalized density profiles of water and ions (total density of cations and anions) for an uncharged surface, with $z$ the distance to the wall. (c) Water enthalpy excess density $\delta h_{\text {wat }}$ profiles, with $z$ the distance to the wall, for different wetting angles $\theta$, controlled by the interaction energy between the liquid and the solid atoms $\varepsilon_{\text {LS. }}$ (d) Water contribution to the thermo-osmotic response coefficient, $M_{\text {to }}^{\text {wat }}$, for different wettings, determined based on water enthalpy excess and slip length computed from MD simulations; stars correspond to $M_{\text {to }}^{\text {wat }}<0$ and circles to $M_{\text {to }}^{\text {wat }}>0$.

sidering the mechanical equilibrium along the $z$ direction, $\frac{\mathrm{d} p}{\mathrm{~d} z}=-\rho_{\mathrm{e}} \frac{\mathrm{d} V}{\mathrm{~d} z}, \delta h_{\mathrm{el}}$ is then usually expressed in terms of the electrostatic potential as:

$$
\delta h_{\mathrm{el}}(z)=-\varepsilon V(z) \frac{\mathrm{d}^{2} V}{\mathrm{~d} z^{2}}+\frac{\varepsilon}{2}\left(\frac{\mathrm{d} V}{\mathrm{~d} z}\right)^{2} .
$$

Accordingly, $\delta h_{\mathrm{el}}$ vanishes outside the EDL, whose thickness is given by the Debye length $\lambda_{\mathrm{D}}$, controlled by the salt concentration. ${ }^{8}$ The corresponding contribution to the thermoosmotic response, $M_{\text {to }}^{\text {el }}$, can be then computed analytically within the mean-field Poisson-Boltzmann framework, ${ }^{37}$ considering a semi-infinite channel (see the ESI $\dagger$ ).

Although the model proposed by Derjaguin et al. can predict $M_{\text {to }}$ experimental orders of magnitude under certain conditions, $^{24}$ it fails to describe the amplitude of the responses reported in the literature, ${ }^{5,16,17,31}$ the thermoosmotic response reported for weakly charged membranes, ${ }^{13}$ 
and the experimental discrepancies observed in $M_{\text {to }}$ sign. ${ }^{21-24}$ Aside from the electrostatic ionic interactions, other contributions to $\delta h$ can be important. Such contributions are related to the solvent (water in the present work), ion solvation, and water dipole orientation in the electric double layer. After comparing all the different contributions to $M_{\text {to }}$ (see the ESI $\dagger$ ), the two main ones are (in the case of symmetric salts such as $\mathrm{NaCl}$ or $\mathrm{KCl})$ :

$$
M_{\mathrm{to}} \simeq M_{\mathrm{to}}^{\mathrm{wat}}+M_{\mathrm{to}}^{\mathrm{e} \mathrm{l}^{*}},
$$

related to the solvent enthalpy excess density $\delta h_{\text {wat }}$ (Fig. 1c and d) and to a modified electrostatic term $\delta h_{\mathrm{el}}^{*}$, accounting for the depletion of the ions in the vicinity of the wall (see the density profiles in Fig. 1b).

Defining the characteristic depletion length as $d_{\ell}$, one can effectively account for this effect by imposing a vanishing enthalpy excess in the interfacial region where there are no ions: $\delta h_{\mathrm{el}}^{*}=\delta h_{\mathrm{el}}$ for $z>d_{\ell}$ and $\delta h_{\mathrm{el}}^{*}=0$ otherwise. With regard to the water contribution, $\delta h_{\text {wat }}$ can be computed as the sum of the different atomic contributions, $\delta h_{\text {wat }}=\delta h_{\mathrm{O}}+\delta h_{\mathrm{H}}$, where the atomic enthalpy density for an element $i$ is defined as:

$$
\delta h_{i}(z)=\left[\delta u_{i}(z)+\delta p_{i}(z)\right] n_{i}(z)
$$

where $\mathrm{d} \mathcal{A}(z)=\mathcal{A}(z)-\mathcal{A}_{\text {bulk }}$, with $\mathcal{A}_{\text {bulk }}$ denoting the bulk value of the physical property $\mathcal{A}, u_{i}$ denoting the energy per atom, $p_{i}$ denoting the stress per atom (a practical difficulty with measuring this term is discussed in the ESI $\dagger$ ), and $n_{i}$ denoting the atomic number density profile.

To compute the solvent term $\delta h_{\text {wat }}$ and the hydrodynamic $\mathrm{BC}$ as a function of wetting, we ran MD simulations using the LAMMPS package. ${ }^{38}$ The system consisted of an aqueous electrolyte (constituted by SPC/E water ${ }^{39}$ and $\mathrm{NaCl}$, such that the bulk salt concentration was $n_{\mathrm{s}} \sim 1 \mathrm{M}$, following ref. 40), confined between generic uncharged Lennard-Jones (LJ) walls or graphene (see Fig. 1b and details in the ESI $\dagger$ ). From these simulations with uncharged walls, we confirmed that in the case of symmetric salts the solute enthalpy, even at large concentrations, did not affect the total enthalpy profile and thus the enthalpy excess density is controlled by the solvent for neutral surfaces (see the ESI $\dagger$ ).

The solid wall atoms were frozen and the oxygen-solid (LS) interactions were varied between the hydrophobic and hydrophilic values given in ref. 40 for LJ walls, corresponding to contact angles on uncharged surfaces of $\theta \sim 140^{\circ}$ and $\theta \sim 50^{\circ}$, respectively (the values for $\theta$ and $b_{\text {eff }}$ can be found in the ESI $\dagger$ ). In Fig. 1c, one can observe the typical shape of the $\delta h_{\text {wat }}$ profile for different wettings. We note that the most hydrophilic situation $\left(\theta \sim 50^{\circ}\right)$ is considered a no-slip situation with $b=0.0 \AA$, corresponding to a stagnant layer $\left(b_{\text {eff }}<0\right)$. For simplicity, we also did not take into account in our model the coupling between the surface charge and slip. ${ }^{41}$ Using the proposed analytical framework, we explored a range of experimentally accessible values for the surface charge density $\Sigma$ and the salt concentration $n_{\mathrm{s}}$ : $\Sigma$ was varied between -1 and
$-300 \mathrm{mC} \mathrm{m}^{-2}$ and $n_{\mathrm{s}} \in\left\{10^{-4}, 1\right\} \mathrm{M}$ corresponding to a Debye length $\lambda_{\mathrm{D}} \in\{0.3,30\} \mathrm{nm}$.

The objective of this communication is to present a general simple model, and in that regard, some approximations are applied in order to explore a broad range of parameters. Nevertheless, the validity of the approximations we use is consistent with the range of parameters we explored, such as the choice of a lower boundary for $\lambda_{\mathrm{D}}$ comparable to the size of water's first absorption layer (where water solvent properties should be accounted for in the calculations, and solvation and water properties should not be considered separately) and the upper boundary for $\Sigma$, under which the mean-field Poisson-Boltzmann description should remain valid ${ }^{37}$ (see the ESI†).

\section{Results and discussion}

From eqn (4), we expect that $M_{\text {to }}$ is controlled by the competition between water and electrostatic contributions, depending on wetting, $\Sigma$ and $n_{\mathrm{s}}$ (or analogously $\lambda_{\mathrm{D}}$ ). In Fig. 2 , the total thermo-osmotic response is represented for all the wettings considered, together with the water contribution $M_{\mathrm{to}}^{\text {wat }}$ (which, by construction, is independent of $\lambda_{\mathrm{D}}$ and $\Sigma$ ) and the modified electrostatic contribution $M_{\text {to }}^{\text {e* }}$ (which is only weakly affected by wetting, mostly through the change in $b_{\text {eff }}$ ). We observed from the figure the rich behavior resulting from that competition, where the water term mostly dominates for the most hydrophobic surfaces $\left(\theta \gtrsim 110^{\circ}\right)$, while for the less hydrophobic surfaces $\left(\theta \lesssim 110^{\circ}\right)$, the electrostatic contribution can dominate for the larger $\lambda_{\mathrm{D}}$. We can also see a large variation in $M_{\text {to }}$ values for different wettings, ranging from $10^{-9}$ to $10^{-7} \mathrm{~m}^{2}$ $\mathrm{s}^{-1}$ for the most hydrophobic case.

A striking result from Fig. 2 is the transition for intermediate wettings from a thermophobic behavior $\left(M_{\mathrm{to}}>0\right)$ at high salt concentrations (small $\left.\lambda_{\mathrm{D}}\right)$ to a thermophilic behavior $\left(M_{\mathrm{to}}\right.$ $<0$ ) at low salt concentrations; see for instance, $\theta \sim 110^{\circ}$. In agreement with previous predictions, ${ }^{24}$ the electrostatic contribution $M_{\mathrm{to}}^{\mathrm{e} \mathrm{l}^{*}}<0$ yields a thermophilic behavior independent of the sign of the surface charge. In contrast, the water term exhibits a change of sign when varying the wetting (see Fig. 1d and the ESI $\dagger$ ). Such change of sign of $M_{\text {to }}^{\text {wat }}$ happens at $\theta \sim 110^{\circ}$ and thus, for $\theta \gtrsim 110^{\circ}, \operatorname{sgn}\left(M_{\mathrm{to}}^{\text {wat }}\right)=-\operatorname{sgn}\left(M_{\mathrm{to}}^{\text {el* }}\right)$ resulting in a change of sign of $M_{\mathrm{to}}$ when $\lambda_{\mathrm{D}}$ is such that $\left|M_{\mathrm{to}}^{\mathrm{wat}}\right|=\left|M_{\mathrm{to}}^{\mathrm{el}^{*}}\right|$. Although this change of behavior happens for all $\theta \gtrsim 110^{\circ}$, for the most hydrophobic cases it takes place for $\lambda_{\mathrm{D}}$ values higher than the ones considered in this study and corresponding to extremely low salt concentrations. Even so, within our parameter range, we can still observe for $\theta \sim 130^{\circ}$ a decrease of the total response for high $\lambda_{\mathrm{D}}$, which goes against the standard expectation and can only happen if water and electrostatic contributions have opposite signs. In contrast, for the less hydrophobic cases $\left(e . g\right.$. $\left.\theta \sim 90^{\circ}\right), \operatorname{sgn}\left(M_{\mathrm{to}}^{\text {wat }}\right)=\operatorname{sgn}\left(M_{\mathrm{to}}^{\mathrm{el}^{*}}\right)$ and $M_{\mathrm{to}}$ does not change sign for any $\lambda_{\mathrm{D}}$ value.

It is interesting to note that a similar change of sign has been found in the context of thermophoresis experiments. ${ }^{42-44}$ 


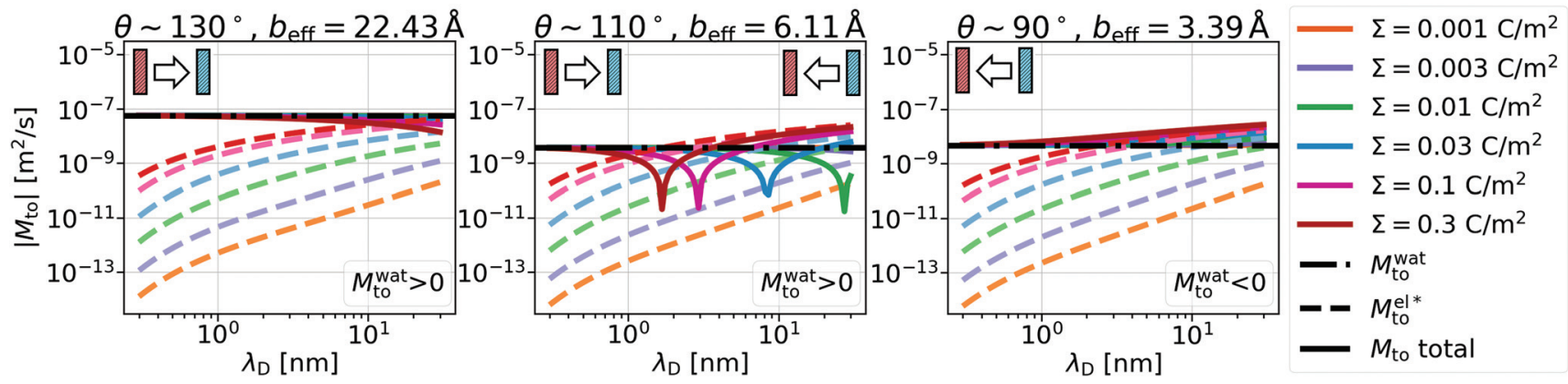

Fig. 2 Thermo-osmotic response coefficient $M_{\text {to }}$ (solid lines) as a function of the Debye length for different wettings and surface charges. In all the graphs, the two main contributions, water $M_{\mathrm{to}}^{\text {wat }}$ (dash-dotted lines) and modified electrostatic $M_{\mathrm{to}}^{\mathrm{e}^{*}}$ (dashed lines), are also represented. While $M_{\mathrm{to}}^{\mathrm{e}{ }^{*}}$ is always negative, the sign of $M_{\text {to }}^{\text {wat }}$ depends on wetting such that the total response can be thermophilic or thermophobic depending on wetting, surface charge and Debye length.

This change of sign is commonly attributed to the so-called thermopotential $\psi_{0} \cdot{ }^{45}$ Such a thermopotential appears when cations and anions have different mobilities and when the channel boundary conditions impose a vanishing flux of each ion type in the bulk. ${ }^{45}$ The thermopotential generates an electro-osmotic flow that can go against the thermo-osmotic flow and reverse the total flow direction. Nevertheless, $\psi_{0}$ should disappear by allowing ionic fluxes through the channel, and as a consequence, the change of sign would disappear. By introducing the water contribution to the thermoosmotic response, we propose a more fundamental understanding of such change of sign, which should persist independently of the boundary conditions on the fluxes through the channel.

The proposed $M_{\mathrm{to}}^{\mathrm{el}}$ and $M_{\mathrm{to}}^{\text {wat }}$ decomposition allows us to obtain an order of magnitude in agreement with the experimental results of $M_{\mathrm{to}}{ }^{24}$ in the order of $10^{-10}-10^{-9} \mathrm{~m}^{2} \mathrm{~s}^{-1}$. Such an agreement is especially significant for hydrophilic surfaces and in the stagnant layer situation (see the ESI $\dagger$ ), typical of experiments due to the presence of imperfections in the solid surface, when $M_{\mathrm{to}}^{\text {wat }}$ decreases and $M_{\mathrm{to}}^{\mathrm{e}{ }^{*}}$ may dominate for a broader range of Debye lengths. Because $M_{\mathrm{to}}^{\text {wat }}$ increases when increasing the slip, one interesting surface is the one constituted by graphene, with an effective slip length of $b_{\text {eff }}=$ $538.77 \AA$, which we obtained in MD simulations for $\mathrm{NaCl}$ aqueous solution at room temperature (see the ESI $\dagger$ ). From the left part of Fig. 3, one can observe a significant increase in both electrostatic and water $M_{\text {to }}$ contributions, resulting in a large value of the total response $\left(M_{\mathrm{to}} \sim 10^{-6} \mathrm{~m}^{2} \mathrm{~s}^{-1}\right)$ for this interface. Because $M_{\mathrm{to}}^{\mathrm{el}}$ does not vary significantly with wetting and the order of magnitude of the total response is given by the water contribution, one can expect $M_{\mathrm{to}} \sim M_{\mathrm{to}}^{\text {wat }}$ for graphene. In the right part of Fig. 3, one can see how $M_{\text {to }}^{\text {wat }}$ is affected by the effective slip. From this figure, one can observe that a large $M_{\text {to }}$ value may be obtained for very slipping systems (as CNT, where slip values of $b \sim 300 \mathrm{~nm}$ have been reported at room temperature for a tube radius of $R \sim 15 \mathrm{~nm}$ (ref. 46)), although it is important to note that the presence of a stagnant layer or defects in the surface (resulting in smaller

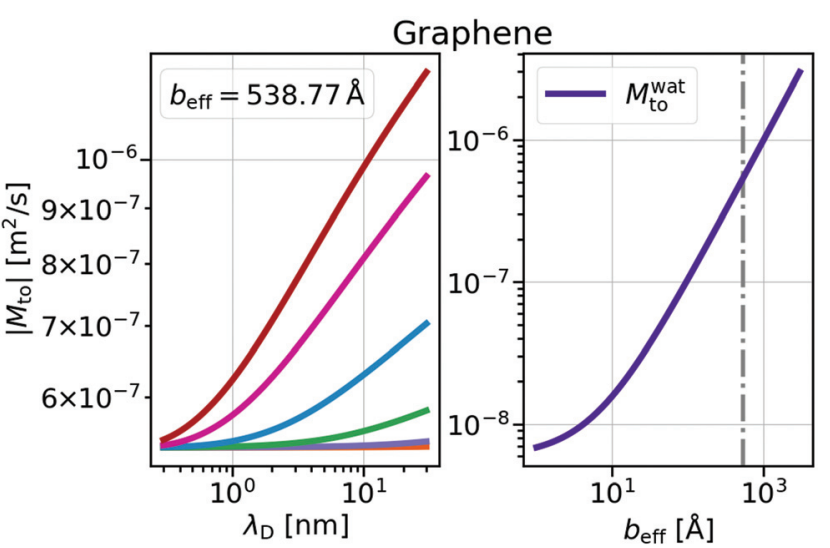

Fig. 3 Thermo-osmotic response coefficient for graphene walls. Left: total response as a function of the Debye length for different surface charges as in Fig. 2. Right: water contribution $M_{\mathrm{to}}^{\text {wat }}$ as a function of the effective slip length. The dash-dotted line indicates the $b_{\text {eff }}$ value obtained from MD simulations. For graphene walls, $M_{\text {to }}^{\text {wat }}$ and $M_{\text {to }}$ are always negative, corresponding to a thermophilic flow.

$\left.b_{\text {eff }}\right)$ may decrease the large predicted thermo-osmotic response, down to $10^{-9} \mathrm{~m}^{2} \mathrm{~s}^{-1}$.

\section{Conclusions}

We proposed here an analytical framework aimed at predicting the thermo-osmotic response of aqueous electrolytes for a wide range of nanofluidic systems and experimental conditions. While the standard picture relates the response to the ion electrostatic enthalpy in the electrical double layer close to charged walls, we show firstly that this contribution to the interfacial enthalpy may be negligible when compared to the water contribution for a broad range of parameters, and secondly that it should be slightly lowered due to the depletion of ions from the solid surface.

The competition between the modified electrostatic and water contributions and the impact of the hydrodynamic 
boundary condition leads to a rich phenomenology that we have illustrated here. First, our theory predicts a higher thermo-osmotic response at low $\lambda_{\mathrm{D}}$ than the one expected from only considering the electrostatic contribution. Second, we predict a thermophobic flow for hydrophobic systems and a thermophilic flow for hydrophilic systems, in agreement with the typical tendencies observed in the experiments. ${ }^{15,25,26}$ Third, for intermediate wettings, the proposed model also predicts a transition between a thermophobic behavior at low salt concentrations to a thermophilic behavior at high salt concentrations. Such a transition has also been observed in thermophoresis experiments ${ }^{42-44}$ and is commonly attributed to the existence of a thermopotential which is, however, limited to particular boundary conditions imposing no ionic fluxes in the bulk liquid. In contrast, our interpretation of the change of sign is more general and independent of the nanofluidic channel boundary conditions, and opens the way to manipulate thermally induced nanoscale flows with a pinch of salt. Finally, we predict intense thermally induced flows for slipping systems, with orders of magnitude comparable to the ones reported from MD simulations of water thermo-osmosis in CNTs ${ }^{5,31}$ or on uncharged planar walls. ${ }^{16,17}$ Such large flows require a significant interfacial enthalpy excess, which we have shown can be obtained for a wide range of wettings, and a large slip length, favored by hydrophobicity. While very hydrophobic materials can pose practical issues in waste heat harvesting with nanoporous membranes, large slip lengths have also been reported for mildly hydrophobic carbon-based materials. ${ }^{46,47}$ Promising hydroelectric energy conversion performance is predicted for such materials, ${ }^{41}$ and our model suggests that thermoelectric energy conversion should also be excellent.

The importance of solvent contribution in thermo-osmosis of aqueous electrolytes, together with a modification of the classical electrostatic term, opens the way to several perspectives. With respect to the electrostatic term, a more accurate description of thermo-osmosis should take into account spatial heterogeneities of the dielectric and viscosity profiles at the interface. ${ }^{48-50}$ In addition, when salt ions adsorb specifically to the surface, e.g. iodide for NaI, the ion-size-dependent hydrophobic solvation term should be considered, e.g. through the modified Poisson-Boltzmann framework described in ref. 40 and 51. Regarding the water term, it is left to determine the impact of the surface charge and its distribution on water contribution to the response. In addition, one could consider more realistic surfaces than the apolar walls described in this study $^{52}$ and, by following the same methodology, we propose to establish the effect of different charge distributions on the total thermo-osmotic response through their effect on the enthalpy excess and on the slip. ${ }^{41}$ Besides, one should take into account the limits of considering pure water simulations as an approximation of the water enthalpy contribution. For high concentrations, steric effects should be accounted for and ions can affect water viscosity. ${ }^{53}$ Nevertheless, such effects correspond to extreme $n_{\mathrm{s}}$ values ${ }^{37}$ and they should not understate one of the main results of this communication: the great
$M_{\text {to }}$ value found for slipping surfaces. Finally, it is straightforward to extend the current model to the Debye-overlap regime (when the system height is smaller than $\lambda_{\mathrm{D}}$ ), as long as the Poisson-Boltzmann framework still holds. In the same line, one can also follow the same procedure here proposed to predict the thermoelectric $c^{6,54,55}$ and thermodiffusive ${ }^{56}$ response, with promising applications for electricity production from waste heat or to refine large-scale continuum descriptions. ${ }^{57}$ Overall, our predictions call for future experimental verification and could be exploited for the design of innovative solutions for heat harvesting applications.

\section{Conflicts of interest}

There are no conflicts to declare.

\section{Acknowledgements}

The authors thank Aymeric Allemand, Anne-Laure Biance, Li $\mathrm{Fu}$ and Christophe Ybert for fruitful discussions. The authors are also grateful for HPC resources from GENCI/TGCC (grants A0070810637 and A0090810637) and the PSMN mesocenter in Lyon. This work is supported by the ANR (Project ANR-16CE06-0004-01 NECtAR). LJ is supported by the Institut Universitaire de France.

\section{References}

1 A. P. Straub, N. Y. Yip, S. Lin, J. Lee and M. Elimelech, Nat. Energy, 2016, 1, 1-6.

2 K. R. Kristiansen, V. M. Barragán and S. Kjelstrup, Phys. Rev. Appl., 2019, 11, 044037.

3 A. P. Straub and M. Elimelech, Environ. Sci. Technol., 2017, 51, 12925-12937.

4 K. Zhao and H. Wu, Nano Lett., 2015, 15, 3664-3668.

5 E. Oyarzua, J. H. Walther, C. M. Megaridis, P. Koumoutsakos and H. A. Zambrano, ACS Nano, 2017, 11, 9997-10002.

6 M. Dietzel and S. Hardt, Phys. Rev. Lett., 2016, 116, 225901.

7 L. Fu, L. Joly and S. Merabia, Phys. Rev. Lett., 2019, 123, 115.

8 T. Markovich, D. Andelman and R. Podgornik, arXiv preprint arXiv:1603.09451, 2016.

9 G. Lippmann, Compt. Rend., 1907, 145, 104-105.

10 M. Aubert, PhD thesis, Verlag nicht ermittelbar, 1912.

11 B. Derjaguin and G. Sidorenkov, C. R. Acad. Sci., 1941, 32, 622-626.

12 M. S. Dariel and O. Kedem, J. Phys. Chem., 1975, 79, 336342 .

13 J. I. Mengual, J. Aguilar and C. Fernandez-Pineda, J. Membr. Sci., 1978, 4, 209-219.

14 R. Piazza, J. Phys.: Condens. Matter, 2004, 16, S4195.

15 V. M. Barragán and S. Kjelstrup, J. Non-Equilib. Thermodyn., 2017, 42, 217-236. 
16 R. Ganti, Y. Liu and D. Frenkel, Phys. Rev. Lett., 2017, 119, 1-5.

17 L. Fu, S. Merabia and L. Joly, Phys. Rev. Lett., 2017, 119, 214501.

18 R. Rajegowda and S. P. Sathian, Phys. Chem. Chem. Phys., 2018, 20, 30321-30330.

19 R. Ganti, Y. Liu and D. Frenkel, Phys. Rev. Lett., 2018, 121, 68002.

20 K. Prakash, K. Dheeraj, S. K. Kannam and S. P. Sathian, Nanotechnology, 2020, 31, 425403.

21 B. Derjaguin, Pure Appl. Chem., 1980, 52, 1163-1178.

22 R. Rusconi, L. Isa and R. Piazza, J. Opt. Soc. Am. B, 2004, 21, 605-616.

23 S. Nedev, S. Carretero-Palacios, P. Kühler, T. Lohmüller, A. S. Urban, L. J. Anderson and J. Feldmann, ACS Photonics, 2015, 2, 491-496.

24 A. P. Bregulla, A. Würger, K. Günther, M. Mertig and F. Cichos, Phys. Rev. Lett., 2016, 116, 1-5.

25 J. Villaluenga, B. Seoane, V. Barragán and C. Ruiz-Bauzá, J. Membr. Sci., 2006, 274, 116-122.

26 S. Kim and M. Mench, J. Membr. Sci., 2009, 328, 113-120.

27 J. Anderson, Annu. Rev. Fluid Mech., 1989, 21, 61-99.

28 B. V. Derjaguin, N. V. Churaev and V. M. Muller, Surface Forces, Springer, 1987.

29 E. Ruckenstein, J. Colloid Interface Sci., 1981, 83, 77-81.

30 R. S. Maheedhara, H. Jing, H. S. Sachar and S. Das, Phys. Chem. Chem. Phys., 2018, 20, 24300-24316.

31 L. Fu, S. Merabia and L. Joly, J. Phys. Chem. Lett., 2018, 9, 2086-2092.

32 C. Navier, Mémoires de l'Académie Royale des Sciences de l'Institut de France, 1823, vol. 6, pp. 389-440.

33 B. Cross, C. Barraud, C. Picard, L. Léger, F. Restagno and É. Charlaix, Phys. Rev. Fluids, 2018, 3, 1-9.

34 C. Herrero, T. Omori, Y. Yamaguchi and L. Joly, J. Chem. Phys., 2019, 151, 041103.

35 L. Bocquet and J.-L. Barrat, Soft Matter, 2007, 3, 685.

36 X. Wang, M. Liu, D. Jing, A. Mohamad and O. Prezhdo, Nano Lett., 2020, 20, 8965-8971.

37 C. Herrero and L. Joly, arXiv preprint arXiv:2105.00720, 2021.
38 S. Plimpton, J. Comput. Phys., 1995, 117, 1-19.

39 H. J. Berendsen, J. R. Grigera and T. P. Straatsma, J. Phys. Chem., 1987, 91, 6269-6271.

40 D. M. Huang, C. Cottin-Bizonne, C. Ybert and L. Bocquet, Phys. Rev. Lett., 2007, 98, 1-4.

41 Y. Xie, L. Fu, T. Niehaus and L. Joly, Phys. Rev. Lett., 2020, 125, 014501.

42 F. S. Gaeta, G. Perna, G. Scala and F. Bellucci, J. Phys. Chem., 1982, 86, 2967-2974.

43 S. A. Putnam and D. G. Cahill, Langmuir, 2005, 21, 53175323.

44 A. Würger, Phys. Rev. Lett., 2008, 101, 108302.

45 A. Würger, Rep. Prog. Phys., 2010, 73, 126601.

46 E. Secchi, S. Marbach, A. Niguès, D. Stein, A. Siria and L. Bocquet, Nature, 2016, 537, 210-213.

47 B. Radha, A. Esfandiar, F. Wang, A. Rooney, K. Gopinadhan, A. Keerthi, A. Mishchenko, A. Janardanan, P. Blake, L. Fumagalli, et al., Nature, 2016, 538, 222-225.

48 H. Hoang and G. Galliero, Phys. Rev. E: Stat., Nonlinear, Soft Matter Phys., 2012, 86, 021202.

49 D. J. Bonthuis and R. R. Netz, J. Phys. Chem. B, 2013, 117, 11397-11413.

50 M. Rezaei, B. G. Mitterwallner, P. Loche, Y. Uematsu, R. R. Netz and D. J. Bonthuis, J. Phys. Chem. B, 2021, 125, 4767-4778.

51 D. M. Huang, C. Cottin-Bizonne, C. Ybert and L. Bocquet, Langmuir, 2008, 24, 1442-1450.

52 C. Wang, H. Lu, Z. Wang, P. Xiu, B. Zhou, G. Zuo, R. Wan, J. Hu and H. Fang, Phys. Rev. Lett., 2009, 103, 137801.

53 J. S. Kim, Z. Wu, A. R. Morrow, A. Yethiraj and A. Yethiraj, J. Phys. Chem. B, 2012, 116, 12007-12013.

54 A. Härtel, M. Janssen, D. Weingarth, V. Presser and R. V. Roij, Energy Environ. Sci., 2015, 8, 2396-2401.

55 Y. Jin, R. Tao, S. Luo and Z. Li, J. Phys. Chem. Lett., 2021, 12, 1144-1149.

56 S. Di Lecce, T. Albrecht and F. Bresme, Nanoscale, 2020, 12, 23626-23635.

57 M. Dietzel and S. Hardt, J. Fluid Mech., 2017, 813, 10601111. 